\title{
Composition of Reflection and Transmission Formulae
}

\author{
John Heading \\ Contribution from the University of Southampton, Southampton, England
}

(Received July 31, 1962)

\begin{abstract}
An integral equation for the electric field in a continuously stratified ionized medium is derived; this is then manipulated to yield equations for the reflection and transmission coefficients, at the same time being susceptible to physical interpretation. The equations are solved by successive approximations, the first terms being Fresnel-type coefficients. Various applications of the results are discussed.
\end{abstract}

\section{Introduction}

In a recent paper, Wait [1962] has followed an idea of Brekhovskikh [1960] and has obtained approximate forms for the reflection coefficients of VLF and ELF radio waves when the ionosphere is not sharply bounded. In the isotropic case, the method is to obtain a first order nonlinear differential equation for $R(z)$, a function of the height $z$ that reduces to the reflection coefficient below the ionized layer. This equation is solved by successive approximations, the first approximation being the familiar Fresnel reflection coefficient. Neither Brekhovskikh in his comprehensive text (p. 220) nor Wait discusses any physical interpretation of the corresponding integral equation. Here, we show that by means of a different, yet ultimately equivalent, formulation of the problem, the resulting equations are susceptible to physical interpretation. The theory is applicable to all angles of incidence and to anisotropic ionospheres. The interpretation is made possible by the recent investigations of Westcott [1962a] in which he has derived various expressions yielding the effect of particular regions of the ionosphere on the reflected wave.

\section{Isotropic Model}

Vertical propagation in an isotropic ionosphere, including the effect of the collision frequency, is governed by the differential equation

$$
\frac{d^{2} E}{d z^{2}}+k^{2}\left(1-\frac{X}{1-i Z}\right) E=0
$$

where the $z$-axis is vertical, $E$ denotes a particular horizontally polarized component of the electric field, $k=\omega / c$ the time factor $e^{i \omega t}$ being suppressed, $X=N e^{2} / \epsilon_{0} m \omega^{2}$, and $Z=\nu / \omega$. We shall write $1-X /(1-i Z)=n^{2}$, where $n$ is the refractive index.

We consider the following configuration for the ionized medium. For $z<a$ there exists free space for which $n=1$; for $z>b$ there exists homogeneous medium extending to infinity for which $X=X_{1}, Z=Z_{1}, n=n_{1}$. For $a<z<b$ the properties of the medium are functions of $z$, varying from $n=1$ to $n=n_{1}$.

Let $\zeta$ denote an intermediate height in the range $a \leq \xi \leq b$. We multiply the equation

$$
E^{\prime \prime}+k^{2} n^{2} E=0
$$

by $e^{-i k n_{1}(z-\zeta)}$ and integrate from $\zeta$ to $b$. To this, we add the result obtained by multiplying by $e^{i k(z-\zeta)}$ and integrating from $a$ to $\zeta$. We have, upon integrating by parts twice in succession, 


$$
\begin{aligned}
\int_{\zeta}^{b} E^{\prime \prime} e^{-i k n_{1}(z-\zeta)} d z=\left.E^{\prime} e^{-i k n_{1}(z-\zeta)}\right|_{\zeta} ^{b}+i k n_{1} \int_{\zeta}^{b} E^{\prime} e^{-i k n_{1}(z-\zeta)} d z & \\
& =\left.\left(E^{\prime}+i k n_{1} E\right) e^{-i k n_{1}(z-\zeta)}\right|_{\zeta} ^{b}-k^{2} n_{1}^{2} \int_{\zeta}^{b} E e^{-i k n_{1}(z-\zeta)} d z .
\end{aligned}
$$

Similarly,

$$
\int_{a}^{\zeta} E^{\prime \prime} e^{i k(z-\zeta)} d z=\left.\left(E^{\prime}-i k E\right) e^{i k(z-\zeta)^{\zeta}}\right|_{a} ^{\zeta}-k^{2} \int_{a}^{\zeta} E e^{i k(z-\zeta)} d z
$$

We now consider a wave incident below the ionosphere. We therefore restrict ourselves to that solution of (1) for which

$$
\begin{aligned}
& E=e^{-i k z}+R e^{i k z} \quad(z \leq a), \\
& E=T e^{-i k n 1^{z}} \quad(z \geq b),
\end{aligned}
$$

$z=0$ being the phase-reference level for both coefficients $R$ and $T$. Then

$$
\left.\left(E^{\prime}+i k n_{1} E\right) e^{-i k n_{1}(z-\zeta)}\right|^{b}=0,
$$

and

$$
\begin{aligned}
\left.\left(E^{\prime}-i k E\right) e^{i k(z-\zeta)}\right|_{a} & =\left(-i k e^{-i k a}+i k R e^{i k a}-i k e^{-i k a}-i k R e^{i k a}\right) e^{i k(a-\zeta)} \\
& =-2 i k e^{-i k \zeta} .
\end{aligned}
$$

The sum of the two integrals (2) and (3) is now given by

$$
\begin{gathered}
\int_{a}^{\zeta} E^{\prime \prime} e^{i k(z-\zeta)} d z+\int_{\zeta}^{b} E^{\prime \prime} e^{-i k n_{1}(z-\zeta)} d z \\
=-i k E(\zeta)-i k n_{1} E(\zeta)+2 i k e^{-i k \zeta}-k^{2} \int_{a}^{\zeta} E e^{i k(z-\zeta)} d z-k^{2} n_{1}^{2} \int_{\zeta}^{b} E e^{-i k n_{1}(z-\zeta)} d z .
\end{gathered}
$$

Hence, when the final term in eq (1) is taken into account, we obtain

$$
i k\left(1+n_{1}\right) E(\zeta)-2 i k e^{-i k \zeta}+k^{2} \int_{a}^{\zeta}\left(1-n^{2}\right) E e^{i k(z-\zeta)} d z+k^{2} \int_{\zeta}^{b}\left(n_{1}^{2}-n^{2}\right) E e^{-i k n_{1}(z-\zeta)} d z=0,
$$

reducing to

$$
E(\zeta)=\frac{2}{1+n_{1}} e^{-i k \zeta}+\frac{i k}{1+n_{1}} \int_{a}^{\zeta} \frac{X}{1-i Z} E(z) e^{i k(z-\zeta)} d z+\frac{i k}{1+n_{1}} \int_{\zeta}^{b}\left(\frac{X}{1-i Z}-\frac{X_{1}}{1-i Z_{1}}\right) E(z) e^{-i k n_{1}(z-\zeta)} d z .
$$

This constitutes a suitable integral equation for the field at any point $\zeta$ in the medium; the integration process is extended over those portions of the ionized medium where the refractive index differs from unity or from $n_{1}$. In particular, if free space exists above $z=b$ (that is, if $n_{1}=1$ ), the integral equation becomes

$$
E(\zeta)=e^{-i k \zeta}+\frac{1}{2} i k \int_{a}^{\zeta} \frac{X}{1-i Z} E(z) e^{i k(z-\zeta)} d z+\frac{1}{2} i k \int_{\zeta}^{b} \frac{X}{1-i Z} E(z) e^{-i k(z-\zeta)} d z .
$$

It should be pointed out that the right-hand sides of both eqs (4) and (5) are independent of $a$, provided that $a$ is any point in the free space below the ionized region.

\section{Reflection and Transmission Formulae}

In eq (4), let $\zeta=a$, in which case 
Then

$$
E(\zeta)=e^{-i k a}+R e^{i k a}
$$

$$
e^{-i k a}+E e^{i k a}=\frac{2}{1+n_{1}} e^{-i k a}+\frac{i k}{1+n_{1}} \int_{a}^{b}\left(\frac{X}{1-i Z}-\frac{X_{1}}{1-i Z_{1}}\right) E(z) e^{-i k n_{1}(z-a)} d z
$$

reducing to

$$
R=\frac{1-n_{1}}{1+n_{1}} e^{-2 i k a}+\frac{i k}{1+n_{1}} e^{-i k\left(1-n_{1}\right) a} \int_{a}^{b}\left(\frac{X}{1-i Z}-\frac{X_{1}}{1-i Z_{1}}\right) E(z) e^{-i k n_{1} z} d z .
$$

In particular, if $n_{1}=1$ (that is, if $X_{1}=0$ ), this reduces to

$$
R=\frac{1}{2} i k \int_{a}^{b} \frac{X}{1-i Z} E(z) e^{-i k z} d z .
$$

It is obvious that the limits may be replaced by $-\infty$ and $+\infty$ respectively, since the integrand vanishes in these extended regions.

Similarly, we place $\zeta=b$ in eq (4), in which case

Hence,

$$
E(\zeta)=T e^{-i k n 1^{b}}
$$

$$
T e^{-i k n_{1} b}=\frac{2}{1+n_{1}} e^{-i k b}+\frac{i k}{1+n_{1}} \int_{a}^{b} \frac{X}{1-i Z} E(z) e^{i k(z-b)} d z,
$$

reducing to

$$
T=\frac{2}{1+n_{1}} e^{-i k\left(1-n_{1}\right) b}+\frac{i k}{1+n_{1}} e^{-i k\left(1-n_{1}\right) b} \int_{a}^{b} \frac{X}{1-i Z} E(z) e^{i k z} d z .
$$

If free space exists above $z=b$, this result simplifies to

$$
T=1+\frac{1}{2} i k \int_{a}^{b} \frac{X}{1-i Z} E(z) e^{i k z} d z .
$$

It should be pointed out that results (6), (7), (8), and (9) are independent of $a$ and $b$ provided that $a$ and $b$ lie in the respective homogeneous regions below and above the ionized medium.

\section{Varying Region Extending to Infinity}

If there exists no homogeneous medium of refractive index $n_{1}$ above $z=b$, then the varying medium will extend to infinity. In particular, the medium becomes infinitely overdense there if $X \rightarrow \infty$ as $z \rightarrow \infty$. Under these circumstances, we multiply eq (1) by $e^{i k(z-\zeta)}$ and integrate from $a$ to $\zeta$ as before, but now we multiply eq (1) by $e^{-i k(z-\zeta)}$ and integrate from $\zeta$ to $\infty$. The same analysis as before leads to the consideration of

$$
\left.\left(E^{\prime}+i k E\right) e^{-i k(z-\zeta)}\right|^{\infty} .
$$

It should be observed that this factor does not converge at the upper limit if a homogeneous medium extends to infinity. It was for this reason that previously we employed the factor $e^{-i k n_{1}(z-\zeta)}$ to secure a result that vanished when $z=b$. For convergence in the present case, we require $E^{\prime}+i k E \rightarrow 0$ as $z \rightarrow \infty$. This limit certainly exists if the region becomes infinitely overdense, since the allowed W.K.B.J. solution for $E$ then becomes exponentially small in magnitude as $z \rightarrow \infty$. We obtain the result

$$
E(\zeta)=e^{-i k \zeta}+\frac{1}{2} i k \int_{a}^{\zeta} \frac{X}{1-i Z} E(z) e^{i k(z-\zeta)} d z+\frac{1}{2} i k \int_{\zeta}^{\infty} \frac{X}{1-i Z} E(z) e^{-i k(z-\zeta)} d z .
$$


We could have multiplied by $e^{-i k n_{1}(z-\zeta)}$ in the second integral, where $n_{1}$ is arbitrary $\left(R l n_{1}>0\right)$, but this would have been an irrelevant procedure in this case since $n_{1}$ would have no definite meaning in the ionized medium.

The formula for $R$ becomes

$$
R=\frac{1}{2} i k \int_{a}^{\infty} \frac{X}{1-i Z} E(z) e^{-i k z} d z
$$

where $a$ occurs below the ionization. No formula for $T$ exists in this case.

\section{Physical Interpretation}

There are three distinct contributions to the field $E(\zeta)$ in eq (4). These are:

(i) $2 e^{-i k \zeta} /\left(1+n_{1}\right)$;

(ii) elements of the form $\frac{i k}{1+n_{1}} \cdot \frac{X_{1}}{1-i Z} E(z) \delta z e^{i k(z-\zeta)}$ originating below the height $\zeta$;

(iii) elements of the form $\frac{i k}{1+n_{1}}\left(\frac{X}{1-i Z}-\frac{X_{1}}{1-i Z_{1}}\right) E(z) \delta z e^{-i k n_{1}(z-\zeta)}$ originating above the height $\zeta$.

The vector sum of all these waves yields the total electric field at the height $\zeta$. (i) is a kind of transmitted wave complete with the appropriate Fresnel transmission coefficient. (ii) represents a plane wave propagated vertically upwards as in free space (according to the factor $e^{-i k \zeta}$ ), originating from the elementary layer of thickness $\delta z$ situated at $z<\zeta$. All such layers below $\zeta$ yield waves of similar forms. (iii) represents a plane wave propagated vertically downwards as in a homogeneous medium of refractive index $n_{1}$ (according to the factor $e^{i k n_{1} \zeta}$ ), originating from the elementary layer of thickness $\delta z$ situated at $z>\zeta$. All such layers above $\zeta$ yield waves of similar forms.

The work of Westcott [1962a] shows that this is the correct interpretation of the elements involved. Each individual layer of thickness $\delta z$ in an ionized medium gives rise to reradiated waves, their originating strength depending on the exact value of the electric field within the layer $\delta z$. In Westcott's series of papers he considered these reradiated waves as propagated in free space, thereby excluding the possibility of a homogeneous medium existing above $z=b$ of refractive index other than unity. His formulae would not converge if such a medium existed there. We have modified the theory to allow for this, at the same time providing the reflection and transmission formulae derived in section 3. It should be observed that our method has not proved the interpretation placed upon the elements involved; to accomplish this would require the more elaborate analysis of Westcott, considering from first principles the Hertz vectors involved in the reradiation processes.

\section{Approximation Methods}

In order to calculate the reflection coefficient (6), it is necessary to know $E(z)$ throughout the medium beforehand. If $E(z)$ were known, $R$ would also be known, implying that there would be no need to evaluate $R$ from formula (6), which after all is but an identity. But an iterative method of solution may be adopted, using approximate solutions derived from eq (4).

A series of approximations may formally be written down based on successive substitution into eq (4). We write

$$
\begin{aligned}
& E_{0}(\zeta)=\frac{2}{1+n_{1}} e^{-i k \zeta} \\
& E_{n}(\zeta)=\frac{i k}{1+n_{1}} \int_{a}^{\zeta} \frac{X}{1-i Z} E_{n-1}(z) e^{i k(z-\zeta)} d z+\frac{i k}{1+n_{1}} \int_{\zeta}^{b}\left(\frac{X}{1-i Z}-\frac{X_{1}}{1-i Z_{1}}\right) E_{n-1}(z) e^{-i k n_{1}(z-\zeta)} d z
\end{aligned}
$$


Now

and if throughout the range $a$ to $b$

$$
\left|E_{0}(\zeta)\right|=\frac{2}{\left|1+n_{1}\right|},
$$

$$
\max \left[\left|\frac{X}{1-i Z}\right|,\left|\left(\frac{X}{1-i Z}-\frac{X_{1}}{1-i Z_{1}}\right) e^{-i k n_{1}(z-\zeta)}\right|\right]=M,
$$

then

$$
\left|E_{n}(\zeta)\right|<\frac{2 k M(b-a)}{\left|1+n_{1}\right|} \max \left|E_{n-1}(z)\right| .
$$

It follows that the series for $E$ converges rapidly provided

$$
\frac{2 k M(b-a)}{\left|1+n_{1}\right|} \ll 1
$$

that is, provided the layer is sufficiently thin $[(b-a)$ small enough] or provided $M$ is small enough (the ionization density small throughout).

If free space exists above $z=b$, the formulae simplify. The successive approximations are

$$
\begin{aligned}
E_{0}(\zeta) & =e^{-i k \zeta} \\
E_{n}(\zeta) & =\frac{1}{2} i k \int_{a}^{\zeta} \frac{X}{1-i Z} E_{n-1}(z) e^{i k(z-\zeta)} d z+\frac{1}{2} i k \int_{\zeta}^{b} \frac{X}{1-i Z} E_{n-1}(z) e^{-i k(z-\zeta)} d z \\
& =\frac{1}{2} i k \int_{a}^{b} \frac{X}{1-i Z} E_{n-1}(z) e^{-i k|z-\zeta|} d z .
\end{aligned}
$$

If $M$ denotes the maximum of $X /|1-i Z|$, the series converges rapidly if

$$
1 / 2 k M(b-a) \ll 1 \text {. }
$$

If $X$ contains a small constant multiplier $\alpha$, evidently this process yields $E(\zeta)$ as a power-series expansion in powers of $\alpha$, since $E_{n}(\zeta) \propto \alpha^{n}$.

The meaning of the individual terms in the series becomes clear. $E_{1}(\zeta)$ represents the reradiated field if the cause of the reradiation process at each level is given by $E_{0}(\zeta)$ and not $E(\zeta)$. Similarly, $E_{2}(\zeta)$ represents the reradiated field due to the field $E_{1}(\zeta)$ only acting on the free electrons, and so on.

Substituting the successive terms of $E(\xi)$ into eq (6) for $R$, we obtain the corresponding series for $R$. The first two terms of (6) become

$$
R=\frac{1-n_{1}}{1+n_{1}} e^{-2 i k a}+\frac{2 i k}{\left(1+n_{1}\right)^{2}} e^{-i k\left(1-n_{1}\right) a} \int_{a}^{b}\left(\frac{X}{1-i Z}-\frac{X_{1}}{1-i Z_{1}}\right) e^{-i k\left(1+n_{1}\right) z} d z .
$$

If $n_{1}=1$ above $z=b$, this reduces to

$$
R_{1}=\frac{1}{2} i k \int_{a}^{b} \frac{X}{1-i Z} e^{-2 i k z} d z
$$

from eq (7). Under these circumstances, eq (9) yields for the first term in $T$,

$$
T=1+\frac{1}{2} i k \int_{a}^{b} \frac{X}{1-i Z} d z .
$$

This value of $R$ arises from the contributions to the reflected wave produced by the incident field $e^{-i k z}$ being regarded as the sole cause of the reradiation process at each level. It corresponds, in fact, to the use of the Born approximation.

For this case, we have from eq (5), 


$$
E_{1}(\zeta)=\frac{1}{2} i k \int_{a}^{b} \frac{X}{1-i Z} e^{-i k z} e^{-i k|z-\zeta|} d z
$$

yielding the second term in the series for $R$ :

$$
R_{2}=\frac{1}{2} i k \int_{a}^{b} \frac{X(z)}{1-i Z(z)} e^{-i k z} d z \cdot \frac{1}{2} i k \int_{a}^{b} \frac{X(t)}{1-i Z(t)} e^{-i k t} e^{-i k|t-z|} d t
$$

With no collisions, we may write this development of $R$ in the form

$$
R=R_{1}+R_{2}=\frac{1}{2} i k \int_{a}^{b} X e^{-2 i k z} d z-\frac{1}{4} k^{2} \int_{a}^{b} X(z) d z\left[e^{-2 i k z} \int_{a}^{z} X(t) d t+\int_{z}^{b} X(t) e^{-2 i k t} d t\right] .
$$

If we place

$$
v=i E^{\prime} / n_{1} k E,
$$

then differentiation and the use of eq (1) show immediately that

where $v=1$ when $z>b$ and

$$
\frac{d v}{d z}=-\frac{i k}{n_{1}}\left(n^{2}-n_{1}^{2} v^{2}\right)
$$

$$
v=\frac{e^{-i k z}-R e^{i k z}}{n_{1}\left(e^{-i k z}+R e^{i k z}\right)}
$$

when $z<a$. This is the equation derived by Wait [1962] and which was solved by successive approximations. The corresponding integral equation would be

$$
v(\zeta)=1+\frac{i k}{n_{1}} \int_{\zeta}^{b}\left(n^{2}-n_{1}^{2} v^{2}\right) d z
$$

no doubt simpler than our eq (5), but certainly less comprehensive in physical content. Moreover, our eq (6) yields $R$ directly as a series expansion, but Wait has shown how eq (13) may be solved approximately, yielding $R$ as a series expansion only indirectly through the expansion of $v$.

\section{Examples}

As the first example, we may consider the model for which free space exists above and below the slab with bounding surfaces $z=0$ and $z=h$; homogeneous medium exists in the slab such that $n^{2}=1-X$. It may easily be shown that the reflection coefficient is given by

$$
R=\frac{\left(n^{2}-1\right)\left(1-e^{2 i k n h}\right)}{(n+1)^{2} e^{2 i k n h}-(n-1)^{2}} .
$$

In order to apply the second-order reflection formula (12), $X$ is assumed to be small within the slab. Expanding $R$ to the second order in $X$, we easily obtain

$$
R=\frac{1}{4} X\left(1-e^{-2 i k h}\right)+\frac{1}{8} X^{2}\left(1-e^{-2 i k h}-2 i k h e^{-2 i k h}\right) .
$$

We may also use eq (12). Since $X$ is constant throughout the range of integration, we obtain

$$
R=\frac{1}{2} i k X \int_{0}^{h} e^{-2 i k z} d z-\frac{1}{4} k^{2} X^{2} \int_{0}^{h} d z\left[e^{-2 i k z} \int_{0}^{z} d t+\int_{z}^{h} e^{-2 i k t} d t\right]
$$

These integrals are trivial to evaluate, the result without any approximation being equal to (14).

Secondly, we may consider a symmetrical Epstein [1930] profile, tending to free-space conditions above and below the layer. Let

$$
X=\frac{\alpha e^{\beta z}}{\left(e^{\beta z}+1\right)^{2}} .
$$


The work of Epstein (or of Budden [1961]) shows that the reflection coefficient has the value

$$
R=\frac{\Gamma(-2 i k / \beta) \Gamma(N+2 i k / \beta) \Gamma(1-N+2 i k / \beta)}{\Gamma(2 i k / \beta) \Gamma(N) \Gamma(1-N)},
$$

where $N$ is given by $N(N-1)+k^{2} \alpha / \beta^{2}=0$. Since $\alpha$ is assumed to be small, we take $N=k^{2} \alpha / \beta^{2}$ approximately, in order to calculate the first term in the development of $R$ in terms of $\alpha$. To this first order, we may neglect the $N$ in the two gamma functions occurring in the numerator. In the denominator we write

$$
\Gamma(N) \Gamma(1-N)=\pi / \sin (\pi N) \fallingdotseq 1 / N
$$

since $N$ is small. Then to the first order

$$
\begin{aligned}
R & =\Gamma(-2 i k / \beta) \Gamma(1+2 i k / \beta) N \\
& =-\frac{\pi N}{\sin (2 i \pi k / \beta)} \\
& =\frac{\pi i \alpha k^{2}}{\beta^{2} \sinh (2 \pi k / \beta)} .
\end{aligned}
$$

This result should also be recovered by the direct application of formula (11). This yields

$$
R=\frac{1}{2} i k \alpha \int_{-\infty}^{\infty} \frac{e^{\beta z} e^{-2 i k z} d z}{\left(e^{\beta z}+1\right)^{2}}
$$

This may be evaluated by considering the corresponding complex contour integral taken around the rectangle with vertices $( \pm L, 0),( \pm L, 2 i \pi / \beta)$, the contour enclosing one double pole at $z=i \pi / \beta$. The result turns out to be identical with (15).

\section{Integral Identities}

The reflection formulae (7) and (10) may be used to obtain certain interesting infinite integrals, when the values of $R, X$, and $E$ are known from other considerations.

Our first illustration involves the exact solution of a tractable model. Consider the exponential profile $X=e^{\alpha z} / k^{2}$ with no collision frequency. The work of Budden [1961] shows that

$$
\begin{gathered}
R=\frac{\Gamma(-2 i k / \alpha)}{\Gamma(2 i k / \alpha)} \alpha^{-4 i k / \alpha}, \\
E(z)=\frac{H_{2 i k / \alpha}^{(1)}\left(2 i e^{\alpha^{z / 2} / \alpha}\right)}{-i \pi^{-1} \Gamma(2 i k / \alpha)\left(e^{i \pi / 2} / \alpha\right)^{-2 i k / \alpha}} .
\end{gathered}
$$

Direct substitution into eq (10) and simplification yield

Finally, let

$$
-2 k \pi^{-1} \Gamma(-2 i k / \alpha) \alpha^{-2 i k / \alpha} e^{\pi k / \alpha}=\int_{-\infty}^{\infty} e^{\alpha z} e^{-i k z} H_{2 i k / \alpha}^{(1)}\left(2 i e^{\alpha z / 2} / \alpha\right) d z .
$$

$$
\nu=2 k / \alpha, t=2 e^{\alpha z / 2} / \alpha,
$$

where $\nu$ is real and positive and arg $t=0$ along the positive real axis. The integral reduces to the result containing the single parameter $\nu$ :

$$
-\nu \pi^{-1} \Gamma(-i \nu) e^{\pi \nu / 2} 2^{1-i_{\nu}}=\int_{0}^{\infty} t^{1-i_{\nu}} H_{i \nu}^{(1)}(i t) d t .
$$

Our second illustration concerns an approximate solution of eq (1). Let $n^{2}$ vary monotonically from 1 when $z=-\infty$ to $-\infty$ when $z=+\infty$, in such a way that $E$ tends to the 
form $e^{-i k z}+R e^{i k z}$ for large negative $z$. Evidently $n^{2}$ possesses a zero at some real value of $z$; we shall suppose that this transition point occurs at the origin 0 . Provided that the value of $k$ is large enough (that is, at the higher frequencies), many authors (see for example [Heading, 1962, page 29]) have shown that an approximate solution for $E$ along the whole real height axis is given by

$$
E=C n^{-1 / 2}\left(\int_{0}^{z} n d z\right)^{1 / 6} \operatorname{Ai}\left[\left(\frac{3}{2} e^{i \pi / 2} k \int_{0}^{z} n d z\right)^{2 / 3}\right]
$$

where Ai denotes the standard Airy integral and $C$ is a constant. This result is a generalization of the simpler W.K.B.J. solutions

$$
n^{-1 / 2} \exp \left( \pm i k \int_{0}^{z} n d z\right)
$$

solutions that cease to be valid near the transition point $z=0$, and that require connection formulae to effect a suitable connection across $z=0$. Equation (16), however, suffers from none of these disadvantages; this solution is valid even at the transition point.

To be specific and consistent, let

$$
\begin{array}{ll}
\arg n=0 & \text { for } z<0, \\
\arg n=-1 / 2 \pi & \text { for } z>0, \\
\arg z=0 & \text { for } z>0, \\
\arg z=\pi & \text { for } z<0 .
\end{array}
$$

When $z>0$, we use the standard asymptotic expression

$$
\text { Ai }(x) \sim \frac{1}{2} \pi^{-1 / 2} x^{-1 / 4} \exp \left(-\frac{2}{3} x^{3 / 2}\right) .
$$

Since $n^{2}$ is negative, we must write $n=e^{-i \pi / 2} m$, where $m>0$; hence

$$
\begin{aligned}
E=C e^{i \pi / 4} m^{-1 / 2}\left(\int_{0}^{z} e^{-i \pi / 2} m d z\right)^{1 / 6} \operatorname{Ai}\left[\left(\frac{3}{2} k \int_{0}^{z} m d z\right)^{2 / 3}\right] & \\
& \sim \frac{1}{2} C \pi^{-1 / 2} e^{i \pi / 6}\left(\frac{3}{2} k\right)^{-1 / 6} m^{-1 / 2} \exp \left(-k \int_{0}^{z} m d z\right),
\end{aligned}
$$

an exponentially evanescent solution as $z \rightarrow \infty$. This solution therefore satisfies the boundary condition there.

When $z<0$, let $z=e^{i \pi} \zeta$, where $\zeta>0$, yielding

$$
\begin{aligned}
E=C n^{-1 / 2}\left(e^{i \pi} \int_{0}^{\zeta} n d \zeta\right)^{1 / 6} \operatorname{Ai}\left[\left(\frac{3}{2} e^{i \pi / 2} k \int_{0}^{\zeta} n e^{i \pi} d \zeta\right)^{2 / 3}\right] & =C n^{-1 / 2} e^{i \pi / 6}\left(\int_{0}^{\zeta} n d \zeta\right)^{1 / 6} \mathrm{Ai}\left[-\left(\frac{3}{2} k \int_{0}^{\zeta} n d \zeta\right)^{2 / 3}\right] .
\end{aligned}
$$

We now quote the standard asymptotic expression

$$
\mathrm{Ai}(-x) \sim \pi^{-1 / 2} x^{-1 / 4} \cos \left(\frac{2}{3} x^{3 / 2}-\frac{1}{4} \pi\right)=\frac{1}{2} \pi^{-1 / 2} x^{-1 / 4}\left[\exp \left(\frac{2}{3} i x^{3 / 2}-\frac{1}{4} \pi i\right)+i \exp \left(-\frac{2}{3} i x^{3 / 2}-\frac{1}{4} \pi i\right)\right],
$$

yielding

$$
\begin{aligned}
E \sim C n^{-1 / 2} e^{i \pi / 6} & \left(\int_{0}^{\zeta} n d \zeta\right)^{1 / 6} \cdot \frac{1}{2} \pi^{-1 / 2}\left(\frac{3}{2} k \int_{0}^{\zeta} n d \zeta\right)^{-1 / 6} \\
& \times\left[\exp \left(i k \int_{0}^{\zeta} n(-\zeta) d \zeta-\frac{1}{4} \pi i\right)+i \exp \left(-i k \int_{0}^{\zeta} n(-\zeta) d \zeta-\frac{1}{4} \pi i\right)\right] \\
& =\frac{1}{2} \pi^{-1 / 2} C e^{i \pi / 6}\left(\frac{3}{2} k\right)^{-1 / 6} n^{-1 / 2}[\exp (\ldots)+i \exp (\ldots)] .
\end{aligned}
$$


In order to introduce the incident and reflected waves, we note that if $\zeta_{1}$ is large and positive, then

$$
\int_{0}^{\zeta} n(-\zeta) d \zeta=\int_{0}^{\zeta_{1}} n(-\zeta) d \zeta+\int_{\zeta_{1}}^{-z} d \zeta
$$

since $n=1$ below $z=-\zeta_{1}$. Hence

$$
\int_{0}^{\zeta} n(-\zeta) d \zeta=-z-\zeta_{1}+\int_{0}^{\zeta_{1}} n(-\zeta) d \zeta=-z+\int_{0}^{\infty}[n(-\zeta)-1] d \zeta,
$$

where the upper limit is replaced by $\infty$ since the integrand must vanish at these additional low heights. It follows that as $n \rightarrow 1$,

$$
\begin{aligned}
E \sim \frac{1}{2} \pi^{-1 / 2} C e^{i \pi / 6}\left(\frac{3}{2} k\right)^{-1 / 6}\left[e^{-i k z} \exp \left(i k \int_{0}^{\infty}[n(-\zeta)-1] d \zeta-\frac{1}{4} \pi i\right)\right. & \\
& \left.+i e^{i k z} \exp \left(-i k \int_{0}^{\infty}[n(-\zeta)-1] d \zeta-\frac{1}{4} \pi i\right)\right] .
\end{aligned}
$$

In order that the incident field should be of unit amplitude, we choose

$$
C^{-1}=\frac{1}{2} \pi^{-1 / 2} e^{i \pi / 6}\left(\frac{3}{2} k\right)^{-1 / 6} \exp \left(i k \int_{0}^{\infty}[n(-\zeta)-1] d \zeta-\frac{1}{4} \pi i\right)
$$

It follows that

$$
\begin{aligned}
& R=i \exp \left(-2 i k \int_{0}^{\infty}[n(-\zeta)-1] d \zeta\right) \\
& X=1-n^{2}, \\
& E=\frac{n^{-1 / 2}\left(\int_{0}^{z} n d z\right)^{1 / 6} \mathrm{Ai}\left[\left(\frac{3}{2} e^{i \pi / 2} k \int_{0}^{z} n d z\right)\right]^{2 / 3}}{\frac{1}{2} \pi^{-1 / 2} e^{i \pi / 6}\left(\frac{3}{2} k\right)^{-1 / 6} \exp \left(i k \int_{0}^{\infty}[n(-\zeta)-1] d \zeta-\frac{1}{4} \pi i\right)} .
\end{aligned}
$$

Final substitution into eq (10) yields after cross multiplication

$$
\begin{aligned}
\frac{1}{2} \pi^{-1 / 2} e^{5 i \pi / 12}\left(\frac{3}{2} k\right)^{-1 / 6} \exp (-i k & \left.\int_{0}^{\infty}[n(-\zeta)--1] d \zeta\right) \\
& =\frac{1}{2} i k \int_{-\infty}^{\infty}\left(1-n^{2}\right) e^{-i k z} n^{-1 / 2}\left(\int_{0}^{z} n d z\right)^{1 / 6} \mathrm{Ai}\left[\left(\frac{3}{2} e^{i \pi / 2} k \int_{0}^{z} n d z\right)^{2 / 3}\right] d z .
\end{aligned}
$$

This formula embraces the buildup of the reflection coefficient from every layer of a slowly varying medium of the type postulated.

\section{Reflection and Transmission Formulae for Anisotropic Ionospheres}

The relevant equations leading to the analysis of this section are given, for example, by Budden [1961, chapter III]. The differential equation for the electric field $\mathbf{E}$ within an anisotropic ionosphere is

$$
\text { curl curl } \mathbf{E}=k^{2} \mathbf{E}+k^{2} \mathbf{M E} \text {, }
$$

where the $3 \times 3$ susceptibility matrix $\mathbf{M}$ is given by

$$
\mathbf{M}=\frac{X}{U\left(U^{2}-Y^{2}\right)}\left(\begin{array}{lll}
-U^{2}+l^{2} Y^{2} & i n Y U+\ln Y^{2} & i m Y U+\ln Y^{2} \\
-i n Y U+\ln Y^{2} & -U^{2}+m^{2} Y^{2} & i l Y U+m n Y^{2} \\
-i m Y U+\ln Y^{2} & -i l Y U+m n Y^{2} & -U^{2}+n^{2} Y^{2}
\end{array}\right) .
$$


Here, $X, Y, Z$ are the usual dimensionless parameters describing the ionosphere, $U=1-i Z$, and $-(l, m, n)$ are the direction cosines of the earth's magnetic field. The vector ME is proportional to the electric polarization vector.

In evaluating curl E, we choose the customary coordinate system in which $\partial / \partial x=-i k$ $\sin \theta, \partial / \partial y=0$, and $\partial / \partial z$ is replaced by a prime. In component form, eq (17) may be written as

$$
\left.\begin{array}{l}
E_{x}^{\prime \prime}+i k \sin \theta E_{z}^{\prime}=-k^{2} E_{x}-k^{2}(\mathbf{M E})_{x}, \\
E_{y}^{\prime \prime}-k^{2} \sin ^{2} \theta E_{y}=-k^{2} E_{y}-k^{2}(\mathbf{M E})_{y}, \\
i k \sin \theta E_{x}^{\prime}-k^{2} \sin \theta E_{z}=-k^{2} E_{z}-k^{2}(\mathbf{M E})_{z} .
\end{array}\right\}
$$

We now multiply these three equations by $e^{-i k z \cos \theta}$ and integrate from $a$ to $b$, namely over the whole height range in which ionization occurs, assuming free space for $z>b$ and $z<a$. Noting that

$$
\begin{aligned}
\int_{a}^{b} E^{\prime} e^{-i k z \cos \theta} d z & =\left.E e^{-i k z \cos \theta}\right|_{a} ^{b}+i k \cos \theta \int_{a}^{b} E e^{-i k z \cos \theta} d z \\
\int_{a}^{b} E^{\prime \prime} e^{-i k z \cos \theta} d z & =\left.\left(E^{\prime}+i k \cos \theta E\right) e^{-i k z \cos \theta}\right|_{a} ^{b}-k^{2} \cos ^{2} \theta \int_{a}^{b} E e^{-i k z \cos \theta} d z,
\end{aligned}
$$

we obtain from eq (18)

$$
\begin{aligned}
& \left.\left(E_{x}^{\prime}+i k \cos \theta E_{x}+i k \sin \theta E_{z}\right) e^{-i k z \cos \theta}\right|_{a} ^{b}=-k^{2} \int_{a}^{b}\left[\sin ^{2} \theta E_{x}+(\mathbf{M E})_{x}-\sin \theta \cos \theta E_{z}\right] e^{-i k z \cos \theta} d z \\
& \left.\left(E_{y}^{\prime}+i k \cos \theta E_{y}\right) e^{-i k z \cos \theta}\right|_{a} ^{b}=-k^{2} \int_{a}^{b}(\mathbf{M E})_{y} e^{-i k z \cos \theta} d z \\
& \left.i k \sin \theta E_{x} e^{-i k z \cos \theta}\right|_{a} ^{b}=-k^{2} \int_{a}^{b}\left[\cos ^{2} \theta E_{z}-\sin \theta \cos \theta E_{x}+(\mathbf{M E})_{z}\right] e^{-i k z \cos \theta}
\end{aligned}
$$

Multiplying the third of these equations by $\tan \theta$ and adding it to the first, we obtain the simpler equation

$$
\left.\left(E_{x}^{\prime}+i k \sec \theta E_{x}+i k \sin \theta E_{z}\right) e^{-i k z \cos \theta}\right|_{a} ^{b}=-k^{2} \int_{a}^{b}\left[(\mathbf{M E})_{x}+\tan \theta(\mathbf{M E})_{z}\right] e^{-i k z \cos \theta} d z
$$

When the incident field is horizontally polarized, let

for $z<a$ and for $z>b$,

$$
\begin{aligned}
& E_{x}=\cos \theta_{\perp} R_{\|} e^{i k z \cos \theta}, \\
& E_{y}=e^{-i k z \cos \theta}+{ }_{\perp} R_{\perp} e^{i k z \cos \theta}, \\
& E_{z}=\sin \theta_{\perp} R_{\|} e^{i k z \cos \theta}
\end{aligned}
$$

$$
\begin{aligned}
& E_{x}=\cos \theta_{\perp} T_{\|} e^{-i k z \cos \theta}, \\
& E_{y}={ }_{\perp} T_{\perp} e^{-i k z \cos \theta}, \\
& E_{z}=-\sin \theta_{\perp} T_{\|} e^{-i k z \cos \theta},
\end{aligned}
$$

the $x$ - and $t$-factors being suppressed. When the incident field is vertically polarized, let

for $z<a$ and for $z>b$,

$$
\begin{aligned}
& E_{x}=\cos \theta e^{-i k z \cos \theta}+\cos \theta_{\|} R_{\|} e^{i k z \cos \theta} \\
& E_{y}={ }_{\|} R_{\perp} e^{i k z \cos \theta} \\
& E_{z}=-\sin \theta e^{-i k z \cos \theta}+\sin \theta_{\|} R_{\|} e^{i k z \cos \theta}
\end{aligned}
$$




$$
\begin{aligned}
& E_{x}=\cos \theta_{\|} T_{\|} e^{-i k z \cos \theta}, \\
& E_{y}={ }_{\Perp} T_{\perp} e^{-i k z \cos \theta}, \\
& E_{z}=-\sin \theta_{\|} T_{\| e^{-i k z \cos \theta} .}
\end{aligned}
$$

Forming an arbitrary linear combination of these two fields, we write

$$
\left.\begin{array}{l}
E_{x}=B \cos \theta e^{-i k z \cos \theta}+\left(A_{\perp} R_{\|}+B_{\|} R_{\|}\right) \cos \theta e^{i k z \cos \theta}, \\
E_{y}=A e^{-i k z \cos \theta}+\left(A_{\perp} R_{\perp}+B_{\|} R_{\perp}\right) e^{i k z \cos \theta}, \\
E_{z}=-B \sin \theta e^{-i k z \cos \theta}+\left(A_{\perp} R_{\|}+B_{\|} R_{\|}\right) \sin \theta e^{i k z \cos \theta}
\end{array}\right\}
$$

for $z<a$ and for $z>b$,

$$
\left.\begin{array}{l}
E_{x}=\left(A_{\perp} T_{\|}+B_{\|} T_{\|}\right) \cos \theta e^{-i k z \cos \theta}, \\
E_{y}=\left(A_{\perp} T_{\perp}+B_{\|} T_{\perp}\right) e^{-i k z \cos \theta}, \\
E_{z}=-\left(A_{\perp} T_{\|}+B_{\|} T_{\|}\right) \sin \theta e^{-i k z \cos \theta} .
\end{array}\right\}
$$

Equation (19) now reduces to

$$
-2 i k \cos \theta\left(A_{\perp} R_{\perp}+B_{\|} R_{\perp}\right)=-k^{2} \int_{a}^{b}(\mathbf{M E})_{y} e^{-i k z \cos \theta} d z,
$$

while (20) takes the form

$$
-2 i k\left(A_{\perp} R_{\|}+B_{\|} R_{\|}\right)=-k^{2} \int_{a}^{b}\left[(\mathbf{M E})_{x}+\tan \theta(\mathbf{M E})_{z}\right] e^{-i k z \cos \theta} d z .
$$

The physical interpretation of these integrals at oblique incidence is demonstrated by considerations given by Westcott, [1962 b, c] and in further papers to be published in this series.

In particular, if the ionized layer is weak so that the field in the medium may be replaced by the incident field as the first approximation, we obtain

$$
\begin{aligned}
A_{\perp} R_{\perp}+B_{\|} R_{\perp}= & -\frac{1}{2} i k \sec \theta \int_{a}^{b}\left(M_{21} B \cos \theta+M_{22} A-M_{23} B \sin \theta\right) e^{-2 i k z \cos \theta} d z, \\
A_{\perp} R_{\|}+B_{\|} R_{\|}= & -\frac{1}{2} i k \int_{a}^{b}\left(M_{11} B \cos \theta+M_{12} A-M_{13} B \sin \theta+M_{31} B \sin \theta\right. \\
& \left.\quad+M_{32} A \tan \theta-M_{33} B \tan \theta \sin \theta\right) e^{-2 \ell k \cos \theta} d z .
\end{aligned}
$$

Hence, if $Z$ is constant, we obtain

$$
\begin{aligned}
& { }_{\perp} R_{\perp}=-\frac{1}{2} i k \sec \theta \int_{a}^{b} M_{22} e^{-2 i k z \cos \theta} d z=\frac{1}{2} i k \sec \theta \frac{U^{2}-m^{2} Y^{2}}{U\left(U^{2}-Y^{2}\right)} \int_{a}^{b} X e^{-2 i k z \cos \theta} d z, \\
& { }_{\|} R_{\perp}=-\frac{1}{2} i k \sec \theta \int_{a}^{b}\left(M_{21} \cos \theta-M_{23} \sin \theta\right) e^{-2 i k z \cos \theta} d z \\
& =\frac{1}{2} i k \sec \theta \frac{\left(i n Y U-l m Y^{2}\right) \cos \theta+\left(i l Y U+m n Y^{2}\right) \sin \theta}{U\left(U^{2}-Y^{2}\right)} \int_{a}^{b} X e^{-2 i k z \cos \theta} d z,
\end{aligned}
$$

${ }_{.} R_{\|}=-\frac{1}{2} i k \int_{a}^{b}\left(M_{12}+M_{32} \tan \theta\right) e^{-2 i k z \cos \theta} d z$

$$
=\frac{1}{2} i k \frac{\left(-i n Y U-l m Y^{2}\right)+\tan \theta\left(i l Y U-m n Y^{2}\right)}{U\left(U^{2}-Y^{2}\right)} \int_{a}^{b} X e^{-2 i k z \cos \theta} d z,
$$

${ }_{\|} R_{\|}=-\frac{1}{2} i k \int_{a}^{b}\left(M_{11} \cos \theta-M_{13} \sin \theta+M_{31} \sin \theta-M_{33} \tan \theta \sin \theta\right) e^{-2 i k z \cos \theta} d z$

$$
=\frac{1}{2} i k \frac{U^{2} \cos 2 \theta \sec \theta-\left(l^{2} \cos \theta-n^{2} \tan \theta \sin \theta\right) Y^{2}-2 i m \sin \theta Y U}{U\left(U^{2}-Y^{2}\right)} \int_{a}^{b} X e^{-2 i k z \cos \theta} d z .
$$


Moreover, we easily deduce the formulae for the isotropic case with $Y=0$ at oblique incidence,

$$
\begin{aligned}
{ }_{\perp} R_{\perp} & =\frac{1}{2} i k \sec \theta U^{-1} \int_{a}^{b} X e^{-2 i k z \cos \theta} d z, \\
{ }_{\|} R_{\|} & =\frac{1}{2} i k \cos 2 \theta \sec \theta U^{-1} \int_{a}^{b} X e^{-2 i k z \cos \theta} d z .
\end{aligned}
$$

The transmission coefficients may be found by multiplying eq (18) by $e^{i k z} \cos \theta$ and integrating over the height range in which ionization occurs. We then multiply the third of the resulting equations by $\tan \theta$ and subtract it from the first, yielding

and

$$
\left.\left(E_{y}^{\prime}-i k \cos \theta E_{y}\right) e^{i k z \cos \theta}\right|_{a} ^{b}=-k^{2} \int_{a}^{b}(\mathbf{M E})_{y} e^{-i k z \cos \theta} d z
$$

$$
\left.\left(E_{x}^{\prime}-i k \sec \theta E_{x}+i k \sin \theta E_{z}\right) e^{i k z \cos \theta}\right|_{a} ^{b}=-k^{2} \int_{a}^{b}\left[(\mathbf{M E})_{x}+\tan \theta(\mathbf{M E})_{z}\right] e^{i k z \cos \theta} d z
$$

Inserting the values (21) and (22) of the field above and below the range of ionization, we obtain

$$
\begin{aligned}
& A_{\perp} T_{\perp}+B_{\|} T_{\perp}=A-\frac{1}{2} i k \sec \theta \int_{a}^{b}(\mathbf{M E})_{y} e^{i k z \cos \theta} d z \\
& A_{\perp} T_{\|}+B_{\|} T_{\|}=B-\frac{1}{2} i k \int_{a}^{b}\left[(\mathbf{M E})_{x}+\tan \theta(\mathbf{M E})_{z}\right] e^{i k z \cos \theta} d z .
\end{aligned}
$$

Finally, we deduce the values of the transmission coefficients to the first order, when the field throughout the medium is replaced by the incident field:

$$
\begin{aligned}
{ }_{\perp} T_{\perp} & =1-\frac{1}{2} i k \sec \theta \int_{a}^{b} M_{22} d z, \\
{ }_{\|} T_{\perp} & =-\frac{1}{2} i k \sec \theta \int_{a}^{b}\left(M_{21} \cos \theta-M_{23} \sin \theta\right) d z, \\
{ }_{\perp} T_{\|} & =-\frac{1}{2} i k \int_{a}^{b}\left(M_{12}+\tan \theta M_{32}\right) d z, \\
{ }_{\|} T_{\|} & =1-\frac{1}{2} i k \int_{a}^{b}\left(M_{11} \cos \theta-M_{13} \sin \theta+M_{31} \sin \theta-M_{33} \tan \theta \sin \theta\right) d z .
\end{aligned}
$$

In the isotropic case, these formulae reduce to

$$
\begin{aligned}
& { }_{\perp} T_{\perp}=1+\frac{1}{2} i k \sec \theta U^{-1} \int_{a}^{b} X d z \\
& T_{\|}=1+\frac{1}{2} i k \cos 2 \theta \sec \theta U^{-1} \int_{a}^{b} X d z .
\end{aligned}
$$

\section{Comparison of the Two Methods}

We should conclude with a note on the difference between the results of Wait [1962] and of the method presented in this paper. Considering the isotropic model at vertical incidence, the first-order reflection coefficient produced here is given by (11), namely

$$
R=\frac{1}{2} i k \int_{a}^{b} X e^{-2 i k z} d z
$$

for zero collision frequency. On the other hand, the first approximation to the solution of the integral eq (13) is obtained by taking $v=1$, yielding 
Hence, when $\xi=a$,

$$
v(\zeta)=1+i k \int_{\zeta}^{b}\left(n^{2}-1\right) d z=1+i k \int_{\zeta}^{b} X d z
$$

or

$$
\frac{e^{-i k a}-R e^{i k a}}{e^{-i k a}+R e^{i k a}}=1+i k \int_{a}^{b} X d z
$$

$$
R=\frac{1}{2} i k e^{-2 i k a} \int_{a}^{b} X d z
$$

when the left hand side is expanded by the binomial since $|R|$ is small.

It is obvious that this result is only valid for a thin layer, in which case the phase factor $e^{-2 i k z}$ in the integrand of (23) is replaced by $e^{-2 i k a}$. Hence, for the homogeneous slab discussed in section 7 , eq (24) yields (when $a=0, b=h$ ),

$$
R=\frac{1}{2} i k \int_{0}^{h} X d z=\frac{1}{2} i k h X .
$$

The exact first term (14) reduces to this only when $k h$ is small. On the other hand, it should be pointed out that Wait's expansion for the homogeneous slab is of a different kind, valid when $|k h \sqrt{ }(1-X)|<<1$.

\section{References}

Brekhovskikh, L. M., Waves in layered media (Academic Press, London and New York, 1960).

Budden, K. G., Radio waves in the ionosphere (Cambridge University Press, England, 1961).

Epstein, P. S., Reflection of waves in an inhomogeneous absorbing medium, Proc. Nat. Acad. Sci. (U.S.A.) 16, $627(1930)$.

Heading, J., An introduction to phase-integral methods (Methuen, London, 1962).

Wait, J. R., On the propagation of VLF and ELF radio waves when the ionosphere is not sharply bounded, J. Research NBS 66D (Radio Prop.) No. 1, 53-61 (Jan.-Feb. 1962).

Westcott, B. S., Ionospheric reflection processes for long radio-waves-I, J. Atmospheric and Terrest. Phys. 24, 385-399 (1962a).

Westcott, B. S., Ionospheric reflection processes for long radio-waves-II, J. Atmospheric and Terrest. Phys. 24, 619-631 (1962b).

Westcott, B. S., Ionospheric reflection processes for long radio-waves-III, J. Atmospheric and Terrest. Phys. 24, 701-713 (1962c).

(Paper 67D1-244) 